\title{
A FORMULA FOR THE INNER SPECTRAL RADIUS
}

\section{S. MAHMOUD MANJEGANI}

Received 3 November 2003

\begin{abstract}
This note presents an asymptotic formula for the minimum of the moduli of the elements in the spectrum of a bounded linear operator acting on Banach space $X$. This minimum moduli is called the inner spectral radius, and the formula established herein is an analogue of Gelfand's spectral radius formula.
\end{abstract}

2000 Mathematics Subject Classification: 47A10.

1. Introduction. Let $X$ be a Banach space and let $B(X)$ denote the Banach algebra of all bounded linear operators on $X$, and let $T$ be an element of $B(X)$. We denote by $m(T)$ the "minimum moduli" of $T$ and define it by

$$
m(T)=\inf \{\|T x\|, x \in X,\|x\|=1\} .
$$

In what follows, $i(T)$ and $i_{a p}(T)$ will denote, respectively, inner spectral radius and inner approximate spectral radius of $T$. We define $i(T)$ and $i_{a p}(T)$ by

$$
\begin{gathered}
i(T)=\inf \{|\lambda|: \lambda \in \sigma(T)\}, \\
i_{a p}(T)=\inf \left\{|\lambda|: \lambda \in \sigma_{a p}(T)\right\} .
\end{gathered}
$$

Makai and Zemanek [3] proved that

$$
i_{a p}(T)=\lim _{n \rightarrow \infty}\left[m\left(T^{n}\right)\right]^{1 / n}
$$

In this note, we prove the same formula for $i(T)$. The main results established herein are the following theorem and corollary.

THEOREM 1.1. Let $T \in B(X)$. Then, $r_{i}(T)=i(T)$ if and only if $r_{i}(T) \leq r_{i}\left(T^{*}\right)$.

COROLlaRY 1.2. It is not necessary that $r_{i}(T)=r_{i}\left(T^{*}\right)$ for any $T \in B(X)$.

2. Basic concepts. Throughout, $X$ will denote a Banach space, $B(X)$ is the Banach algebra of all bounded linear operators on $X$. $T$ will denote an element of $B(X)$. We denote $T^{*}$ as the transpose of $T\left(T^{*}\right.$ is an element of $\mathrm{B}\left(X^{*}\right)$, where $X^{*}$ is dual space of $X)$ and define

$$
\left(T^{*} g\right)(x)=g(T(x)), \quad x \in X, g \in X^{*} .
$$


If $X$ is a Hilbert space, then $T^{*}$ is the adjoint of $T$ and $T^{*} \in B(X)$. We denote by $\sigma(T)$, $\sigma_{a p}(T), \sigma_{p}(T)$, and $\sigma_{c}(T)$, respectively, spectrum, approximate point spectrum, point spectrum, and compression spectrum of $T$ and define

$$
\begin{aligned}
\sigma(T) & =\{\lambda:(T-\lambda I) \text { is not invertible, } \lambda \in \mathbb{C}\}, \\
\sigma_{a p}(T) & =\{\lambda:(T-\lambda I) \text { is not bounded below, } \lambda \in \mathbb{C}\}, \\
\sigma_{p}(T) & =\{\lambda: \operatorname{ker}(T-\lambda I) \neq 0, \lambda \in \mathbb{C}\}, \\
\sigma_{\mathcal{c}}(T) & =\{\lambda: \operatorname{ran}(T-\lambda I) \text { is not dense in } X, \lambda \in \mathbb{C}\} .
\end{aligned}
$$

The spectral radius of $T$ is denoted by $r(T)$ and defined by

$$
r(T)=\sup \{|\lambda|: \lambda \in \sigma(T)\}
$$

We recall the following statements. One can see their proof in [1].

(1) $\|T\|=\left\|T^{*}\right\|$.

(2) $r(T)=\lim _{n \rightarrow \infty}\left\|T^{n}\right\|^{1 / n}$ (Gelfand's formula).

(3) $r(T)=r\left(T^{*}\right)$.

(4) $\sigma(T)=\sigma\left(T^{*}\right)$, if $X$ is a Hilbert space, then $\sigma\left(T^{*}\right)=\overline{\sigma(T)}$, where $\overline{\sigma(T)}=\{\bar{\lambda}, \lambda \in$ $\sigma(T)\}$.

For operator $T \in B(X)$, define

$$
m(T)=\inf \{\|T x\|, x \in X,\|x\|=1\}
$$

$m(T)$ is called the minimum moduli of $T$. Note that by definition of $m(T)$, we have $\|T x\| \geq m(T)\|x\|$. It is clear that; if $T$ is an invertible element in $B(X)$, then $m(T)=$ $\left\|T^{-1}\right\|^{-1}$.

DEFINITION 2.1. The inner spectral radius and inner approximate spectral radius of $T$ are denoted, respectively, by $i(T)$ and $i_{a p}(T)$ and defined by (1.2).

Proposition 2.2. If $|\lambda|<m(T)$, then $(T-\lambda I)$ is bounded below.

Proof. We have

$$
\|(T-\lambda I) x\| \geq\|T x\|-\|\lambda x\| \geq(m(T)-|\lambda|)\|x\| .
$$

The assumption implies that $m(T)-|\lambda|>0$ and hence $(T-\lambda I)$ is bounded below by the definition.

Proposition 2.3. For every operator $T \in B(X)$,

$$
\lim _{n \rightarrow \infty}\left[m\left(T^{n}\right)\right]^{1 / n}=\sup \left[m\left(T^{n}\right)\right]^{1 / n} .
$$


Proof. For every operator $T$ and $S$ in $B(X)$, we have

$$
m(T S) \geq m(T) m(S)
$$

by definition of the minimum moduli. Therefore, for every positive integers $i$ and $j$,

$$
m\left(T^{i+j}\right) \geq m\left(T^{i}\right) m\left(T^{j}\right) .
$$

This is the crucial inequality. Let $k$ be fixed. For every integer number $n$, we have $n=k q+r, 0 \leq r<k$, where $q=q(n)$ and $r=r(n)$ are functions of $n$. Note that $\lim _{n \rightarrow \infty} q(n) / n=1 / k$. Thus, by (2.8) we have

$$
m\left(T^{n}\right) \geq m\left(T^{k}\right)^{q} m(T)^{r}, \quad \text { for each positive integer } n \text {. }
$$

Hence,

$$
\lim _{n \rightarrow \infty} \inf \left[m\left(T^{n}\right)\right]^{n} \geq m\left(T^{k}\right)^{1 / k}
$$

Since this equation holds for all $k$, we have

$$
\lim _{n \rightarrow \infty} \inf \left[m\left(T^{n}\right)\right]^{n} \geq \sup \left[m\left(T^{n}\right)\right]^{1 / n} \geq \lim _{n \rightarrow \infty} \sup \left[m\left(T^{n}\right)\right]^{n}
$$

and the result follows.

Assume that $r_{i}(T)=\lim _{n \rightarrow \infty}\left[m\left(T^{n}\right)\right]^{1 / n}$. By Gelfand's formula, it is clear that if $T$ is invertible, then $r_{i}(T)=\left[r\left(T^{-1}\right)\right]^{-1}$.

Corollary 2.4. Let $T \in B(X)$. Then, $0 \in \sigma_{a p}(T)$ if and only if $m(T)=0$.

Proof. The result follows from the facts that $0 \in \sigma_{a p}(T)$ if and only if $T$ is not bounded below and $\|T x\| \geq m(T)\|x\|$ for each $x \in X$.

Proposition 2.5. Let $T \in B(X)$. If $\lambda \in \sigma_{a p}(T)$, then $|\lambda| \geq r_{i}(T)$.

Proof. Suppose $\lambda \in \sigma_{a p}(T)$. Assume, contrary to what we wish to prove, that $|\lambda|<r_{i}(T)$. Thus, $|\lambda|^{n}<m\left(T^{n}\right)$ for some integer $n$ by the definition of $r_{i}(T)$. By Proposition 2.2, $\left(T^{n}-\lambda^{n} I\right)$ is bounded below. We have

$$
T^{n}-\lambda^{n}=\left(T^{n-1}+T^{n-2} \lambda+\cdots+\lambda^{n}\right)(T-\lambda) .
$$

Hence, $(T-\lambda)$ is bounded below and so $\lambda \notin \sigma_{a p}(T)$, which is contradictory to our assumption. 
COROLlary 2.6. For each $T \in B(X)$,

$$
\sigma_{a p}(T) \subseteq\left\{\lambda: r_{i}(T) \leq|\lambda| \leq r(T)\right\}
$$

Makai and Zemanek in [3] proved that $i_{a p}(T)=r_{i}(T)$ for every $T \in B(X)$. In the next section, we will prove that $i(T)=r_{i}(T)$ if and only if $r_{i}(T) \leq r_{i}\left(T^{*}\right)$.

3. Inner spectral radius. The purpose of this section is to prove the main result. We know that $\partial \sigma_{a p}(T) \subseteq \sigma(T)$ and $r_{i}(T)=i_{a p}(T)$ and so $r_{i}(T) \in \sigma(T)$. Therefore, for every $T \in B(X)$, we have

$$
i(T) \leq r_{i}(T) .
$$

FACT 3.1. If $X$ is a finite-dimensional space, then $\sigma_{a p}(T)=\sigma(T)$ for each $T \in B(X)$ and hence $r_{i}(T)=i(T)$.

FACT 3.2. If $T$ is a compact operator acting on Banach space $X$, then $r_{i}(T)=i(T)$.

We begin with some general lemmas that we need in the proof of the main theorem.

LEMmA 3.3. Let $T \in B(X)$. Then, $\sigma_{c}(T)=\sigma_{p}\left(T^{*}\right)$. (If $X$ is a Hilbert space, then $\left.\sigma_{c}(T)=\overline{\sigma_{p}\left(T^{*}\right)}\right)$.

Proof. First, we show that $\sigma_{c}(T) \subseteq \sigma_{p}\left(T^{*}\right)$. Suppose $\lambda$ is an element in $\sigma_{c}(T)$. Consider $M$ the closure of $\operatorname{ran}(T-\lambda I)$. By definition of $\sigma_{c}(T), M \neq X$. If $x_{0}$ is a nonzero element in $X-M$, then by the Hahn-Banach theorem there is $f_{0} \in X^{*}$ such that $f_{0}(M)=0$ and $f_{0}\left(x_{0}\right)=1$. We have $\left(\left(T^{*}-\lambda I\right) f_{0}\right)(x)=f_{0}((T-\lambda I) x)=0$ for every $x \in X$ and hence $f_{0} \in \operatorname{ker}\left(T^{*}-\lambda I\right)$, that is $\lambda \in \sigma_{p}\left(T^{*}\right)$.

Now, we prove $\sigma_{p}\left(T^{*}\right) \subseteq \sigma_{c}(T)$. Suppose $\lambda \in \sigma_{p}\left(T^{*}\right)$, thus, there is a nonzero functional $g$ in $X^{*}$ such that $\left(T^{*}-\lambda I\right) g=0$ and so, $g((T-\lambda I) x)=0$ for each $x \in X$ by (2.1). Hence, $g(t)=0$ for any $t$ in closure $\operatorname{ran}(T-\lambda I)$.

But $g \neq 0$ on $X$, and hence there is $x_{0} \in X-M$ such that $g\left(x_{0}\right) \neq 0$. Therefore, $M \neq X$, that is, $\lambda \in \sigma_{c}(T)$.

If $X$ is a Hilbert space, then we know that $\operatorname{ker}(T)=\left(\operatorname{ran} T^{*}\right)^{\perp}$ and $\operatorname{closure}\left(\operatorname{ran} T^{*}\right)=$ $(\operatorname{ker} T)^{\perp}$ in [1, Theorem II.2.19]. Thus, by the definition of $\sigma_{p}(T)$ and $\sigma_{c}(T)$, we get the following result.

LEMmA 3.4. Let $T \in B(X)$. Then, $\sigma(T)=\sigma_{a p}(T) \bigcup \sigma_{c}(T)$.

Proof. It follows from [1, Proposition VII.6.4] and the definition of $\sigma_{a p}(T)$ and $\sigma_{c}(T)$.

LEMmA 3.5. Let $T \in B(X)$. If $\sigma(T) \subseteq\left\{\lambda: r_{i}(T) \leq|\lambda| \leq r(T)\right\}$, then $r_{i}(T)=i(T)$.

Proof. By assumption, we have $r_{i}(T) \leq i(T)$ and the result follows the fact that $i(T) \leq r_{i}(T)$.

THEOREM 3.6. Let $T \in B(X)$. Then, $r_{i}(T)=i(T)$ if and only if $r_{i}(T) \leq r_{i}\left(T^{*}\right)$. 
Proof. First, suppose that $r_{i}(T) \leq r_{i}\left(T^{*}\right)$. By Lemmas 3.3 and 3.4, $\sigma(T)=\sigma_{a p}(T) \bigcup$ $\sigma_{p}\left(T^{*}\right)$ (if $X$ is a Hilbert space, then $\sigma(T)=\sigma_{a p}(T) \cup \overline{\sigma_{p}\left(T^{*}\right)}$ ). We have

$$
\sigma(T) \subseteq\left\{\lambda: r_{i}(T) \leq|\lambda| \leq r(T)\right\} .
$$

Hence, by Lemma 3.5, $r_{i}(T)=i(T)$.

Conversely, suppose that $r_{i}(T)=i(T)$. We have $\sigma(T)=\sigma\left(T^{*}\right)$ (if $X$ is a Hilbert space, then $\left.\sigma\left(T^{*}\right)=\overline{\sigma(T)}\right)$. Thus, $i(T)=i\left(T^{*}\right)$ by definition of $i(T)$, and, therefore,

$$
r_{i}(T)=i(T)=i\left(T^{*}\right) \leq r_{i}\left(T^{*}\right) .
$$

EXAMPLE 3.7. Let $X$ be a Hilbert space and $N \in B(X)$ a normal operator. Then,

$$
i(N)=i\left(N^{*}\right)=r_{i}(N)=r_{i}\left(N^{*}\right) .
$$

Since $N$ is normal, $\|N x\|=\left\|N^{*} x\right\|$ for every $x$ in $X$, and, therefore, $m(N)=m\left(N^{*}\right)$. Similarly, we have $m\left(N^{n}\right)=m\left(N^{* n}\right)$ for each $n$, and so, $i(N)=i\left(N^{*}\right)=r_{i}(N)=r_{i}\left(N^{*}\right)$.

If $X$ is a Hilbert space and $N \in B(X)$ is a normal operator, then $r(N)=\|N\|$. In the next proposition, we prove that $r_{i}(N)=m(N)$ for the normal operator $N$ in $B(X)$.

Recall that for each operator $T \in B(X)$ the numerical range of $T$ is defined and denoted as follows:

$$
W(T)=\{\lambda \in \mathbb{C}: \lambda=\langle T x, x\rangle, x \in X \text { with }\|x\|=1\} .
$$

The following interesting theorem was proved in [2, Theorem 27.9].

THEOREM 3.8. If $T$ is a selfadjoint operator in $B(X), M_{1}$ and $M_{2}$ denote, respectively, the infimum and the supremum of the numerical range of $T$, then $M_{1}$ and $M_{2}$ are approximate eigenvalues of $T$, and the spectrum of $T$ is contained in the interval $\left[M_{1}, M_{2}\right]$.

By this theorem, for each positive operator $T \in B(X)$ we have

$$
i(T)=r_{i}(T)=\inf \{\langle T x, x\rangle, x \in X \text { with }\|x\|=1\} .
$$

Proposition 3.9. If $N$ is normal operator acting on Hilbert space $X$, then $i(N)=$ $r_{i}(N)=m(N)$.

Proof. As shown in Example 3.7, we have $m(N)=m\left(N^{*}\right)$. Now, we prove that $m\left(N N^{*}\right)=m(N)^{2}$. Since $N N^{*}$ is positive, by (3.6) and Proposition 2.3, we have

$$
\begin{aligned}
m\left(N N^{*}\right) \leq r_{i}\left(N N^{*}\right) & =\inf \left\{\left\langle N N^{*} x, x\right\rangle, x \in X \text { with }\|x\|=1\right\} \\
& =\inf \left\{\|N x\|^{2}, x \in X \text { with }\|x\|=1\right\}=m(N)^{2} .
\end{aligned}
$$

By (2.8), we get

$$
m\left(N N^{*}\right) \geq m(N) m\left(N^{*}\right)=m(N)^{2} .
$$

Hence,

$$
m\left(N N^{*}\right)=m(N)^{2} .
$$


By induction, we show that if $j=2^{n}, n=0,1,2, \ldots$, then $m\left(N^{j}\right)=m(N)^{j}$. This is clearly true for $n=0$. Assume it to be true for some $n$, then for all $x \in \mathfrak{n}$, we have

$$
\left\|N^{2^{n+1}}(x)\right\|=\left\|N^{2^{n}}\left(N^{2^{n}}(x)\right)\right\|=\left\|\left(N^{2^{n}}\right)^{*}\left(N^{2^{n}}(x)\right)\right\|,
$$

because $N^{2^{n}}$ is normal. This shows that $m\left(N^{2^{n+1}}\right)=m\left(\left(N^{2^{n}}\right)^{*} N^{2^{n}}\right)$, which is equal to $m\left(N^{2^{n}}\right)^{2}$. Thus, $m\left(N^{2^{n+1}}\right)=\left(m(N)^{2^{n}}\right)^{2}=m(N)^{2^{n+1}}$. Therefore,

$$
r_{i}(N)=\lim _{n \rightarrow \infty}\left[m\left(N^{n}\right)\right]^{1 / n}=\lim _{n \rightarrow \infty}\left[m\left(N^{2^{n}}\right)\right]^{1 / 2^{n}}=m(N) .
$$

EXAMPLE 3.10. Suppose $U$ is a unilateral weighted shift with weights $(1,2,1, \ldots)$ acting on separable Hilbert space $\tilde{6}$. William Ridge [4] proved that $\sigma_{a p}(U)=\{\lambda:|\lambda|=\sqrt{ } 2\}$, $\sigma(U)=\{\lambda:|\lambda| \leq \sqrt{ } 2\}$, and $\sigma_{a p}\left(U^{*}\right)=\sigma\left(U^{*}\right)=\sigma(U)$. Hence, $r_{i}(U)=r(U)=\sqrt{ } 2$, $r_{i}\left(U^{*}\right)=i\left(U^{*}\right)=0$, and $i(U)=0$. Therefore, we have $i(U) \neq r_{i}(U)$ and $r_{i}\left(U^{*}\right)<r_{i}(U)$.

We know that $r(T)=r\left(T^{*}\right)$ for any $T \in B(X)$. But in the above example $r_{i}\left(U^{*}\right)<$ $r_{i}(U)$ so, we can write the next corollary.

Corollary 3.11. It is not necessary that $r_{i}(T)=r_{i}\left(T^{*}\right)$ for any $T \in B(X)$.

ACKNOWLEDGments. The author wishes to thank Professors Douglas R. Farenick and Juliana Erlijman for their advice and helpful comments regarding the results herein. This work is supported by the Isfahan University of Technology, Iran, and the University of Regina, Canada.

\section{REFERENCES}

[1] J. B. Conway, A Course in Functional Analysis, 2nd ed., Graduate Texts in Mathematics, vol. 96, Springer-Verlag, New York, 1990.

[2] B. V. Limaye, Functional Analysis, Wiley Eastern, New Delhi, 1981.

[3] E. Makai, Jr. and J. Zemánek, The surjectivity radius, packing numbers and boundedness below of linear operators, Integral Equations Operator Theory 6 (1983), no. 3, 372384.

[4] W. C. Ridge, Approximate point spectrum of a weighted shift, Trans. Amer. Math. Soc. 147 (1970), 349-356.

S. Mahmoud Manjegani: School of Mathematical Science, Isfahan University of Technology, Isfahan 84156, Iran

E-mail address: manjgani@cc . iut.ac. ir

Current address: Department of Mathematics and Statistics, University of Regina, Regina, Saskatchewan, Canada S4S 0A2

E-mail address: manjegan@math.uregina.ca 


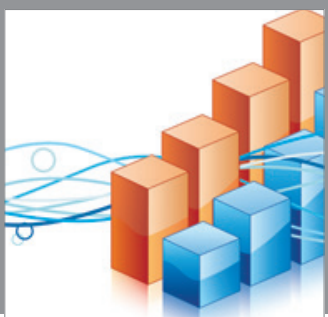

Advances in

Operations Research

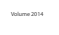

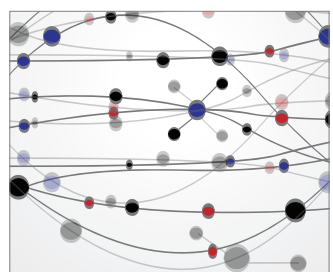

\section{The Scientific} World Journal
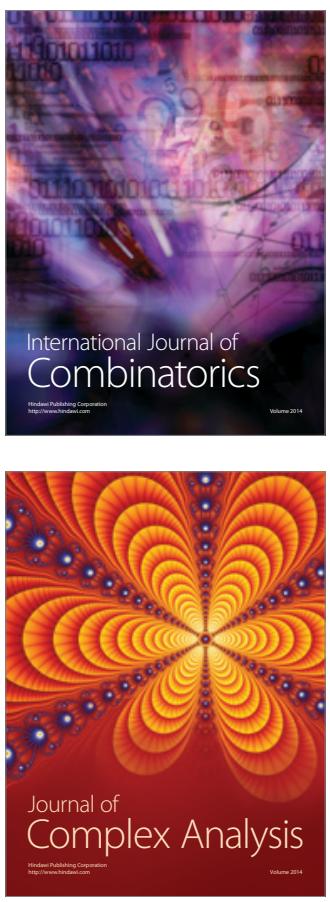

International Journal of

Mathematics and

Mathematical

Sciences
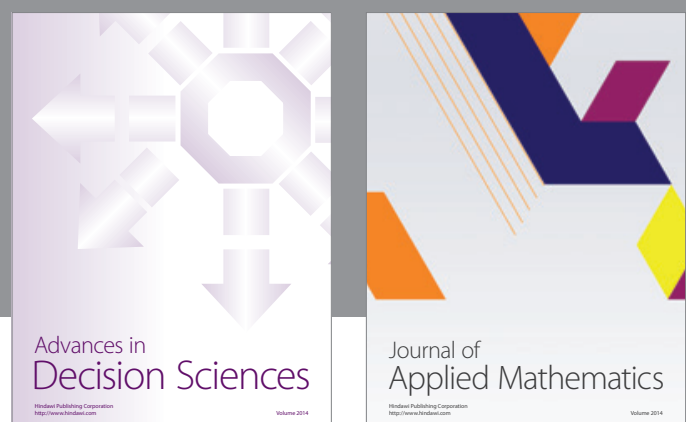

Journal of

Applied Mathematics
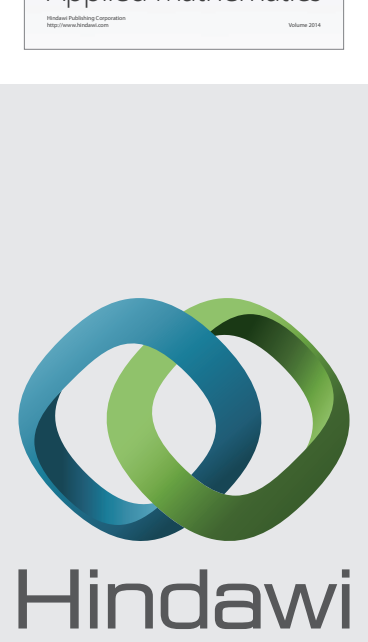

Submit your manuscripts at http://www.hindawi.com
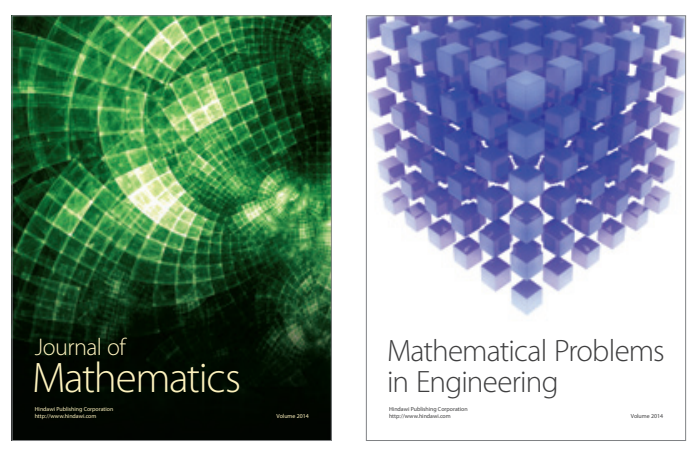

Mathematical Problems in Engineering
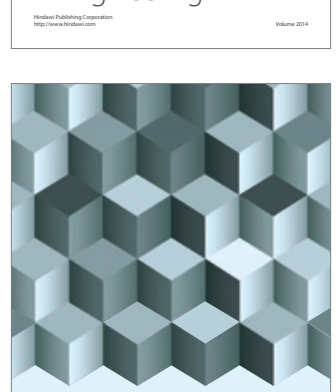

Journal of

Function Spaces
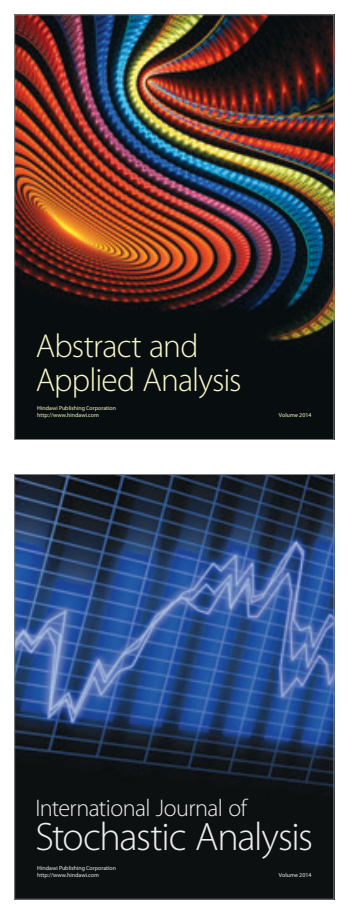

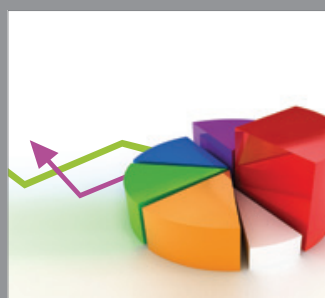

ournal of

Probability and Statistics

Promensencen
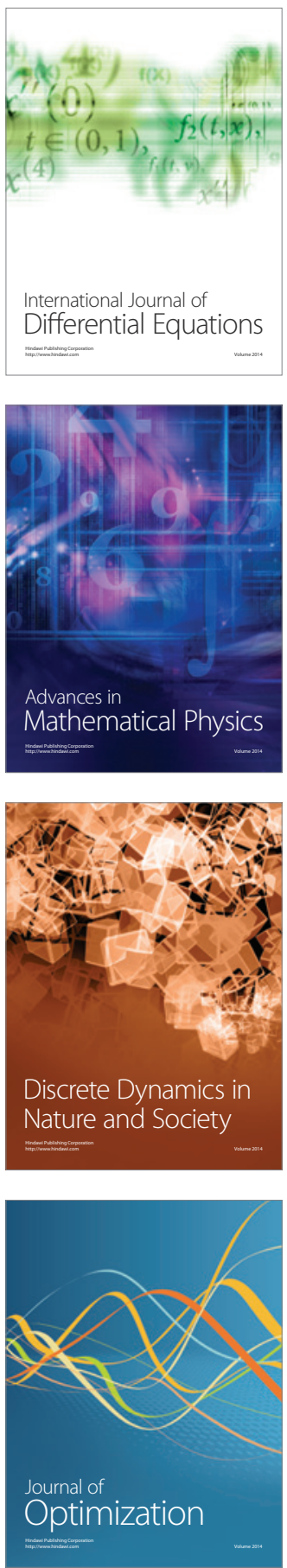